\title{
BMJ Open The FOOTPATH study: protocol for a multicentre, participant- and assessor- blind, parallel group randomised clinical trial of foot orthoses for patellofemoral osteoarthritis
}

\author{
Natalie J Collins, ${ }^{\circledR 1,2}$ Jade M Tan, ${ }^{2,3}$ Hylton B Menz, ${ }^{2,3}$ Trevor G Russell, ${ }^{1}$ \\ Anne J Smith, ${ }^{4}$ Bill Vicenzino, ${ }^{1}$ Shannon E Munteanu, ${ }^{2,3}$ Rana S Hinman, ${ }^{5}$ \\ Terry P Haines, ${ }^{6}$ Harvi F Hart, ${ }^{2,7}$ Brooke E Patterson, ${ }^{2}$ Gearoid Cleary, ${ }^{1}$ \\ Joel W Donnar, ${ }^{2}$ Liam R Maclachlan, ${ }^{1}$ Kay M Crossley ${ }^{2}$
}

To cite: Collins NJ, Tan JM, Menz HB, et al. The FOOTPATH study: protocol for a multicentre, participant- and assessor-blind, parallel group randomised clinical trial of foot orthoses for patellofemoral osteoarthritis. BMJ Open 2019;9:e025315. doi:10.1136/ bmjopen-2018-025315

\section{- Prepublication history and} additional material for this paper are available online. To view these files, please visit the journal online (http://dx.doi. org/10.1136/bmjopen-2018025315).

Received 18 July 2018 Revised 20 February 2019 Accepted 21 February 2019

Check for updates

(C) Author(s) (or their employer(s)) 2019. Re-use permitted under CC BY-NC. No commercial re-use. See rights and permissions. Published by BMJ.

For numbered affiliations see end of article.

Correspondence to Dr Natalie J Collins; n.collins1@uq.edu.au

\section{ABSTRACT}

Introduction Patellofemoral (PF) osteoarthritis (OA) is a common and burdensome subgroup of knee $0 \mathrm{~A}$, with very little evidence for effective treatments. Prefabricated foot orthoses are an affordable and accessible intervention that have been shown to reduce PF pain in younger adults. Similarities between PF pain and PFOA, as well as our pilot work, suggest that foot orthoses may also be an effective intervention for PFOA. The primary objective of this study is to compare the 3 month efficacy of prefabricated foot orthoses and flat shoe inserts in people with PFOA, on knee pain severity.

Methods and analysis The FOOTPATH Study (F0ot OrThoses for PAtellofemoral osteoarTHritis) is a multicentre, randomised, participant- and assessorblinded superiority trial with two parallel groups, a 3 month observation period (pre-randomisation) and 12 month follow-up. 160 participants with a clinical diagnosis of PFOA will be recruited from three sites in Australia, and randomised to one of two groups (prefabricated foot orthoses or flat shoe inserts). The primary outcome is worst knee pain severity during a self-nominated aggravating activity in the previous week (100 mm visual analogue scale) at 3 months, with a secondary endpoint at 12 months. Secondary outcomes include global rating of change, symptoms, function, health-related quality of life, kinesiophobia, self-efficacy and use of co-interventions for knee pain. Blinded, intention-to-treat analyses of primary and secondary patient-reported outcomes will be performed, as well as economic analyses.

Ethics and dissemination Ethical approval has been granted by La Trobe University's Human Ethics Committee and The University of Queensland's Medical Research Ethics Committee. Study outcomes will be disseminated via peer-reviewed journals, conference presentations targeting a range of healthcare disciplines and an open access website with clinician resources. Trial registration number ANZCTRN12617000385347; Pre-results.

\section{Strengths and limitations of this study}

- This multicentre study is the first full-scale randomised clinical trial to evaluate simple, prefabricated foot orthoses as a treatment for patellofemoral osteoarthritis.

- The proposed project will recruit a large sample of people with patellofemoral osteoarthritis, with sample size estimates based on our pilot work.

- Outcomes will be measured at 3 months (primary endpoint), as well as 12 months to evaluate the longer-term efficacy of foot orthoses for this chronic condition.

- Economic analyses will provide cost-effectiveness ratios and costs per additional quality-adjusted life year, to inform clinical decision-making.

- While participants and outcome assessors are blinded, it is not possible to blind the therapists issuing the interventions, due to visual differences between the prefabricated foot orthoses and flat shoe inserts.

\section{INTRODUCTION}

Patellofemoral (PF) osteoarthritis (OA) is an important subgroup of knee OA, whose burden is becoming increasingly evident. Radiographic PFOA is more common than tibiofemoral (TF) OA in people with chronic knee pain (64\% to $69 \%$ compared with $44 \%$ to $45 \%) .{ }^{12}$ The PF joint is often the first knee joint compartment affected by $\mathrm{OA}$, and increases the risk of TFOA development and progression. ${ }^{3}$ Structural features of PFOA show greater association with knee symptoms than TFOA features. Patellofemoral osteophytes (but not TF osteophytes) are associated with knee pain (OR 2.3, 95\% CI 1.1 to $4.8),{ }^{4}$ and reduced patellar cartilage volume (but not femoral or tibial) is related to greater 
pain and functional impairment. ${ }^{5}$ Importantly, compared with TFOA, PFOA tends to occur in younger people, ${ }^{1}$ who often have greater daily physical demands due to occupational and/or childcare responsibilities. Considering the progressive nature of PFOA, the side effects of long-term medication use, and that pain and functional limitations are primary barriers to physical activity ${ }^{6}$ and indications for total knee replacement, ${ }^{7}$ interventions that can effectively reduce PFOA pain are urgently required.

Despite the burden of PFOA, and best-practice guidelines recommending non-surgical, non-drug interventions as the first line strategy for knee OA management, ${ }^{7}$ there is very little evidence for effective treatments for PFOA. Although combined interventions (eg, PF taping, knee/hip exercises, manual therapy, education) ${ }^{89}$ and knee braces ${ }^{10}$ have some evidence of efficacy, their longer-term effects appear to be limited by poor treatment adherence. ${ }^{11} 12$ This is particularly relevant for middle-aged adults with PFOA, whose busy lifestyles and family and work commitments are likely to influence adherence to exercise programmes. ${ }^{11}$ Issues with knee brace bulkiness and interference with clothing ${ }^{12}$ are likely to be barriers to brace wear. For braces and orthoses to be effective, they must be comfortable and unobtrusive to daily living to ensure maximal adherence and patient outcomes.

Foot orthoses are inserts worn in everyday footwear that are contoured to match the shape of the foot. Prefabricated foot orthoses are affordable and accessible, and are an effective treatment for PF pain in young adults (aged 18 to 40 years). ${ }^{1314}$ Based on similarities in symptoms, biomechanics and muscle function between PF pain and PFOA ${ }^{15-17}$ it is plausible that foot orthoses could also have positive effects in people with PFOA. Pilot data show that people with PFOA ( $\mathrm{n}=23$, mean age $59 \pm 10$ years) report immediate improvements in pain when performing a step-down task with foot orthoses, compared with shoes alone. ${ }^{18}$ We observed high adherence and only transient, minor adverse events in our previous trial of foot orthoses in PF pain, ${ }^{19}$ suggesting the feasibility of long-term wear. It is therefore timely to conduct a randomised clinical trial (RCT) to evaluate foot orthoses efficacy in this population. The FOOTPATH Study (FOot OrThoses for PAtellofemoral osteoarTHritis) will investigate the efficacy of prefabricated foot orthoses for people with PFOA.

\section{OBJECTIVES}

\section{Primary objective}

The primary objective is to compare the 3 month efficacy of prefabricated foot orthoses and flat shoe inserts on knee pain severity in people with PFOA. We hypothesise that, compared with flat inserts, foot orthoses will result in greater improvements in knee pain during a nominated aggravating activity at 3 months (H1).

\section{Key secondary objectives}

1. Compare the 3 month efficacy of prefabricated foot orthoses and flat shoe inserts in people with PFOA, on patient-reported global rating of change (GROC).
We hypothesise that, compared with flat inserts, foot orthoses will result in more participants reporting marked improvement at 3 months (H2).

2. Compare the 12 month efficacy of prefabricated foot orthoses and flat shoe inserts on GROC, knee pain severity, function, quality of life, kinesiophobia, self-efficacy and use of co-interventions, in people with PFOA. We hypothesise that foot orthoses will yield: (i) more participants reporting marked improvement, and greater improvements in knee pain during a nominated aggravating activity, at 12 months (H3); and (ii) greater improvements in knee pain severity, the Knee injury and Osteoarthritis Outcome Score (KOOS), Anterior Knee Pain Scale, Short-Form 12, EuroQol-5D, Tampa Scale for Kinesiophobia and Arthritis Self Efficacy Scale, and less co-intervention use at 3 and 12 months (H4).

3. Evaluate the 12 month economic efficiency of prefabricated foot orthoses compared with flat shoe inserts in people with PFOA.

We hypothesise that foot orthoses will yield better cost-effectiveness ratios and lower costs per additional quality-adjusted life year after 12 months (H5).

\section{Other secondary objectives}

Alongside primary and secondary RCT outcomes, we will investigate the following additional secondary objectives, in people with $\mathrm{PFOA}$.

1. Identify factors that predict change in patient-reported symptoms over a 3 month wait-and-see period.

2. Describe characteristics of people with PFOA, including patterns of pain location.

3. Investigate whether foot mobility is related to radiographic features of $\mathrm{PF}$ and TF joint alignment and radiographic features of $\mathrm{OA}$.

4. Identify clinically applicable factors that predict poor prognosis at 3 and 12 months, and determine baseline values of predictor variables to facilitate clinical identification of people with a poor prognosis.

5. Determine the 3 month effect of prefabricated foot orthoses on physical activity level compared with flat shoe inserts.

6. Explore factors that are associated with clinical outcomes with prefabricated foot orthoses at 3 and 12 months.

\section{METHODS AND ANALYSIS}

\section{Trial design}

The FOOTPATH Study is a multicentre, randomised, participant- and assessor-blinded superiority trial with two parallel groups, a 3 month observation period (pre-randomisation) and 12 month follow-up. Equal numbers of participants will be randomised to each group, with the primary endpoint of GROC and pain after 3 months. The trial will be conducted across two university sites in Melbourne and Brisbane, Australia, with a satellite site in Hobart, Tasmania. The trial protocol was developed in consultation with the Standard Protocol Items: 
Recommendations for Interventional Trials (SPIRIT) Statement ${ }^{20} 21$ and the Osteoarthritis Research Society International (OARSI) recommendations. ${ }^{22}$ The trial was prospectively registered.

\section{Ethics approval}

In the event that a substantive modification to the study protocol is required (ie, modifications that affect the conduct of the study), a formal protocol amendment will be prepared and all proposed amendments reviewed by the two ethics committees. These will be reported in the Australian New Zealand Clinical Trials Registry and study publications.

\section{Participant recruitment and eligibility criteria}

Figure 1 summarises the flow of participants through the study. Participants will be recruited from the community in Melbourne, Brisbane, Hobart and regional Victoria. We will use a multifaceted recruitment strategy that has successfully recruited people of all ages with knee pain in our previous studies. This will include strategies such as paid and free advertisements in local newspapers, community magazines and newsletters (eg, university staff bulletins, seniors newsletters); posters in senior citizen's centres, golf and bowling clubs and retirement villages; sandwich boards and handouts at community events (eg,

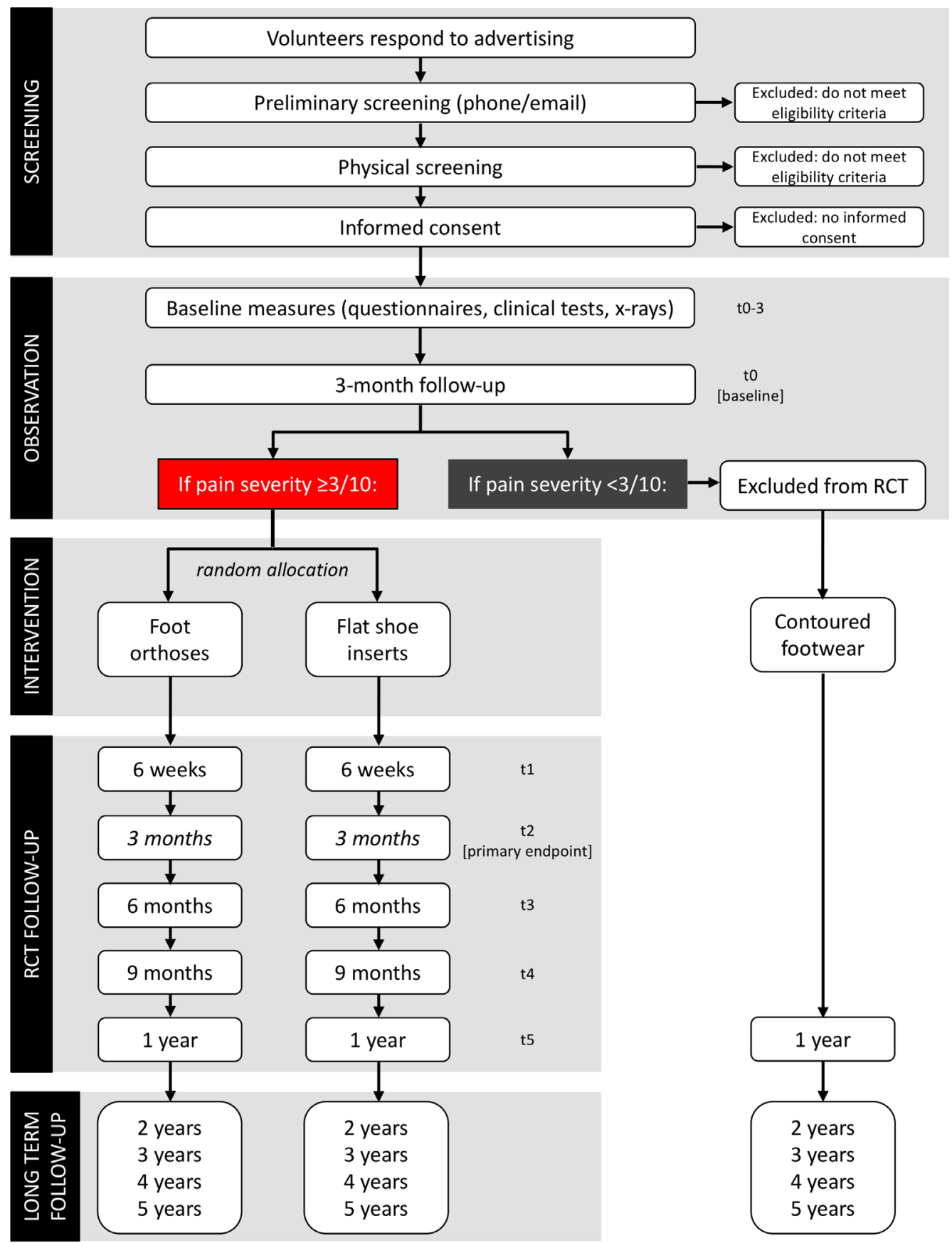

Figure 1 Flow of participants through the study. RCT, randomised clinical trial. 
fun runs, farmer's markets); radio and television media releases; mail-outs to health practitioners in recruitment areas (eg, general practitioners, orthopaedic surgeons, physiotherapists); posts on university and research centre websites (La Trobe University, The University of Queensland); social media (eg, Facebook, Twitter) and patients from the La Trobe University Health Sciences (Podiatry) Clinic, community healthcare centres and hospital waiting lists. Based on recruitment rates of six participants per month during our feasibility trial (single site), as well as known periods of slow recruitment (eg, December/January), conservative estimates indicate that the duration of recruitment will be approximately 20 months. Recruitment rates across all sites will be monitored during the trial, and recruitment strategies adjusted accordingly to meet recruitment targets. There will be no incentives provided to trial investigators or participants for enrolment.

Volunteers who respond to advertisements will be screened for eligibility using a two-stage screening process. This will be conducted by an experienced musculoskeletal health professional (physiotherapist or podiatrist with a minimum of 5 years of musculoskeletal clinical experience). Preliminary screening questions will be asked via telephone or email. Potentially suitable volunteers will then be invited to attend a physical screening appointment at La Trobe University, The University of Queensland or a private practice (if in regional Victoria or Hobart), where a comprehensive musculoskeletal examination will be completed.

We will use a clinical diagnosis of PFOA, ${ }^{23}$ adapted from the National Institute for Health and Care Excellence (NICE) guidelines. ${ }^{24}$ This is to facilitate generalisation of findings to clinical practice, without the need for imaging. Inclusion criteria will be: (1) age 50 years and over, (2) predominant symptom of anterior or retropatellar knee pain aggravated by at least two PF joint loading activities (eg, stairs, squatting, rising from sitting), (3) pain present during these activities on most days of the previous month, (4) pain severity of at least three on an 11-point numerical rating scale $(0-10)$ during aggravating activities, (5) duration of symptoms of at least 3 months and (6) either no morning joint-related stiffness or morning stiffness that lasts no longer than $30 \mathrm{~min}$.

Volunteers will be excluded if they have: (1) knee pain symptoms predominantly from other knee (TF joint) structures, hip or lumbar spine, (2) knee injections or use of any shoe inserts within the previous 3 months, (3) recent commencement of new physiotherapy treatment for $\mathrm{PF}$ pain (ie, new intervention or modifications to existing intervention such as therapeutic exercise), (4) any foot condition precluding the use of foot orthoses or flat shoe inserts, (5) history of lower limb surgery involving major reconstructive procedure (eg, anterior cruciate ligament reconstruction, osteotomy, arthroplasty), (6) planned lower limb surgery in the following 12 months, (7) neurological or systemic arthritis conditions, (8) major medical conditions (eg, cancer), (9) contraindications to X-ray (pregnancy, breastfeeding) or (10) an inability to understand written and spoken English.

\section{Informed consent}

All volunteers who meet the study eligibility criteria will be provided with a participant information sheet. This will provide details of the first phase of the study (observation period) and outline procedures for the second phase of the study (intervention). A trained investigator will discuss the study with volunteers and provide opportunities for volunteers to ask any questions. The lead investigator at each university site (Melbourne: $\mathrm{KMC}$ and Brisbane: NJC) will be available for consultation as required. All participants will provide written informed consent prior to participation. At the conclusion of the observation period, participants will provide additional consent for the intervention phase of the study (detailed below). Participant information and consent forms for all components of the study are included in online supplementary file 1 .

\section{Baseline assessment}

Participants will attend a single session at La Trobe University, The University of Queensland or a private physiotherapy/podiatry clinic (if in regional Victoria or Hobart) for baseline assessment. Structured questionnaires and established patient-reported outcome measures will be used. These will be administered in an electronic format (via computer or tablet) to familiarise participants with the electronic platform. Participants will then nominate their preferred methods of communication (eg, phone, email) and questionnaire completion (paper or electronic format ${ }^{25}$ ) for the duration of the study.

Participant characteristics will include age, sex, occupation, duration of knee pain symptoms, major medical conditions, other joint complaints in the past month ${ }^{26}$ and medication use.

The patient-reported outcome measures are outlined in figure 2 and detailed in online supplementary file 2. Pain will be evaluated as knee pain severity over the past week $(100 \mathrm{~mm} \text { visual analogue scale [VAS] })^{27}$ with painDETECT $^{28}$ and Navigate Pain. ${ }^{29}$ The KOOS ${ }^{30}$ and patellofemoral subscale ${ }^{31}$ will evaluate pain severity, other symptoms, function, knee-related quality of life and patellofemoral symptoms. Other measures include the Anterior Knee Pain Scale (AKPS), ${ }^{32}$ Short-Form-12 (SF-12) questionnaire, ${ }^{33}$ EuroQol (EQ) 5D-5L questionnaire, ${ }^{34}$ Tampa Scale for Kinesiophobia, ${ }^{35}$ Pain Catastrophising Scale, ${ }^{36}$ Arthritis Self Efficacy Scale ${ }^{37}$ and sport and physical activity participation.

Participants will also complete a battery of clinical measures and tests (detailed in online supplementary file 3), which were selected based on their potential to predict PFOA prognosis and/or response to foot orthoses. These include height, mass, body mass index (BMI), waist circumference, presence of knee clicking and crepitus, ${ }^{38}$ Foot Posture Index,${ }^{39}$ foot mobility (foot assessment platform),${ }^{40}$ weight-bearing ankle dorsiflexion 


\begin{tabular}{|c|c|c|c|c|c|c|c|}
\hline \multirow[b]{3}{*}{ TIMEPOINT* } & \multicolumn{7}{|c|}{ STUDY PERIOD } \\
\hline & \multicolumn{2}{|c|}{$\begin{array}{l}\text { Enrolment / } \\
\text { Baseline }\end{array}$} & \multicolumn{4}{|c|}{ Post-allocation } & \multirow{2}{*}{$\begin{array}{c}\begin{array}{c}\text { Close } \\
\text { out }\end{array} \\
\text { t5 }\end{array}$} \\
\hline & t0-3 & to & t1 & t2 & $\mathrm{t} 3$ & t4 & \\
\hline \multicolumn{8}{|l|}{ ENROLMENT: } \\
\hline Eligibility screen & $x$ & & & & & & \\
\hline Informed consent & $x$ & $x$ & & & & & \\
\hline Allocation & & $x$ & & & & & \\
\hline \multicolumn{8}{|l|}{ INTERVENTIONS: } \\
\hline Contoured shoe inserts & & $\curvearrowleft$ & & & & & $\longrightarrow$ \\
\hline Flat shoe inserts & & $\hookleftarrow$ & & & & & $\longrightarrow$ \\
\hline \multicolumn{8}{|l|}{ ASSESSMENTS: } \\
\hline Participant characteristics & $x$ & & & & & & \\
\hline Knee pain severity & $x$ & & & & & & \\
\hline Navigate Pain & $x$ & & & & & & \\
\hline PainDetect & $x$ & & & & & & \\
\hline Pain Catastrophising Scale (PCS) & $x$ & & & & & & \\
\hline Sport and physical activity participation & $x$ & & & & & & \\
\hline Anthropometric measures & $x$ & & & & & & \\
\hline Knee clicking and crepitus & $x$ & & & & & & \\
\hline Knee extension torque & $x$ & & & & & & \\
\hline Foot Posture Index (FPI) & $x$ & & & & & & \\
\hline Foot mobility & $x$ & & & & & & \\
\hline Weight bearing ankle dorsiflexion range of motion & $x$ & & & & & & \\
\hline Footwear Assessment Tool & $x$ & & & & & & \\
\hline 10-metre Walk Test & $x$ & & & & & & \\
\hline \multicolumn{8}{|l|}{ Primary outcome measure: } \\
\hline $\begin{array}{l}\text { Worst knee pain severity during self-nominated } \\
\text { aggravating activity in the previous week }\end{array}$ & $x$ & $x$ & $x$ & $x$ & $x$ & $x$ & $x$ \\
\hline \multicolumn{8}{|l|}{ Secondary outcome measures: } \\
\hline Patient-reported global rating of change (GROC) & & & $x$ & $x$ & & & $x$ \\
\hline Pain visual analogue scales & $x$ & $x$ & $x$ & $x$ & $x$ & $x$ & $x$ \\
\hline Knee injury and Osteoarthritis Outcome Score (KOOS) & $x$ & $x$ & & $x$ & & & $x$ \\
\hline Anterior Knee Pain Scale (AKPS) & $x$ & $x$ & & $x$ & & & $x$ \\
\hline Arthritis Self-Efficacy Scale (ASES) & $x$ & $x$ & & $x$ & & & $x$ \\
\hline Tampa Scale for Kinesiophobia (TSK) & $x$ & $x$ & & $x$ & & & $x$ \\
\hline Short-form 12 (SF-12) & $\mathrm{x}$ & $x$ & & $x$ & & & $\mathrm{x}$ \\
\hline Euroqol-5D-5L (EQ-5D) & $x$ & $x$ & & $x$ & $x$ & $x$ & $x$ \\
\hline \multicolumn{8}{|l|}{ Other outcomes: } \\
\hline Use of co-interventions for knee pain & & & & $x$ & $x$ & $x$ & $x$ \\
\hline Adverse events & & & & $x$ & $\mathrm{x}$ & $x$ & $x$ \\
\hline Direct health care costs & & & & $x$ & $x$ & $x$ & $x$ \\
\hline iMTA Productivity Cost Questionnaire (iPCQ) & & & & $x$ & $x$ & $x$ & $x$ \\
\hline Credibility and Expectancy Questionnaire (CEQ) & & $x$ & & $\mathrm{x}$ & & & $\mathrm{x}$ \\
\hline
\end{tabular}

Figure 2 Standard Protocol Items: Recommendations for Interventional Trials (SPIRIT) diagram of enrolment, interventions and assessments for the FOot OrThoses for PAtellofemoral osteoarTHritis (FOOTPATH) study.

(knee to wall test) ${ }^{41}$ Footwear Assessment Tool, ${ }^{42}$ knee extension torque ${ }^{43}$ and the timed 10 -metre walk test. ${ }^{44}$

\section{Radiographic assessment}

All participants will attend a private radiology clinic to have radiographs taken of their nominated study knee (most symptomatic eligible knee if pain is bilateral). These will be used to characterise the cohort and used as predictor variables in other secondary analyses. Several radiology clinics in Melbourne, regional Victoria, Hobart and Brisbane will be used to minimise participant travel time. Weight-bearing anteroposterior, lateral and skyline views will be obtained using standard clinical protocols. Radiographs will be used to grade the presence and severity of $\mathrm{OA}$ features in the PF and TF joint compartments. Radiographic features of joint space narrowing and osteophytes will be graded and the presence of $\mathrm{PF}$ and TF OA determined using the Kellgren-Lawrence grading system ${ }^{45}$ and a radiographic atlas. ${ }^{46}$ Each 
radiograph will be graded by two investigators (NJC, JMT, KMC), including at least one experienced investigator. Anteroposterior radiographs will also be used to measure frontal plane TF alignment, ${ }^{47}$ while lateral and skyline views will be used to measure PF alignment using established protocols. ${ }^{48}$

\section{Observation period}

Participants will undergo a 3 month observation period, where they will not receive any treatment for their knee pain as part of the study. This is to ensure that only participants with ongoing chronic symptoms that do not improve with time are enrolled in the RCT. Participants will be informed that they will be observed for a 3 month period before receiving their intervention.

During the observation period, a subgroup of participants will undergo physical activity monitoring. This subgroup will consist of the first 60 participants who have access to the internet and a smartphone or laptop and who agree to participate. They will be asked to wear a Fitbit device (Flex/Flex 2, Fitbit Inc., San Francisco, USA) for the duration of the 3 month observation period. This is to familiarise participants with the device and to facilitate adherence with wear during the next phase of the study. Data for physical activity will be remotely extracted from the Fitbit website.

To maintain contact during the observation period, participants will be contacted via phone or email 6 weeks after their baseline measures, and will be asked to rate their average and worst knee pain severity over the past week during their nominated aggravating activity (11-point numerical rating scale). The patient-reported outcome measures taken at baseline will be repeated 3 months after initial assessment. Participants who rate their pain during aggravating activities as less than $30 \mathrm{~mm}$ on a $100 \mathrm{~mm}$ VAS will not be invited to participate in the RCT. They will be offered a pair of contoured sandals (Vionic, Arundel, Queensland, Australia), and be invited to participate in a prospective longitudinal cohort study (a separate consent process). The same battery of questionnaires administered at baseline will be completed at yearly intervals from the date of baseline assessment (up to 5years), with the addition of questionnaires regarding GROC, use of co-interventions for knee pain and adverse events. This study will occur alongside, but separate to, the RCT.

Participants who rate their worst knee pain at least $30 \mathrm{~mm}$ on the $100 \mathrm{~mm}$ VAS during their nominated aggravating activity will be invited to participate in the RCT evaluating the efficacy of prefabricated foot orthoses, compared with flat shoe inserts.

\section{Randomised clinical trial \\ Informed consent}

Participants who are eligible to participate in the RCT will provide separate informed consent for the RCT and for the release of their Medicare and Pharmaceutical Benefits Scheme data for economic analyses.
RCT baseline measures

Three-month follow-up outcomes from the observation period will serve as baseline data for the RCT. Participants will also complete the Credibility and Expectancy Questionnaire (CEQ) to evaluate treatment expectations. ${ }^{49}$

\section{Allocation, concealment and blinding}

Once baseline outcome measures are completed, participants will be randomised to receive prefabricated foot orthoses or flat shoe inserts. To ensure concealed allocation, we will use an offsite, telephone-based interactive voice response randomisation service (National Health and Medical Research Council Clinical Trials Centre, randomisation will be performed using a computer-generated minimisation programme with study site as a minimisation factor). Each participant's allocated intervention will be revealed to a single investigator (JMT), who will communicate this to the participant's nominated study practitioner or to the Brisbane site research assistant (GC) who will liaise with local study practitioners. Because we are comparing two shoe inserts with different shapes, it is not possible to blind study practitioners to group allocation. As the primary outcomes are self-reported, participants are considered assessors. To ensure participant (and thus assessor) blinding, consent will involve limited disclosure. As in our recent RCT, ${ }^{8}$ participants will be informed that they will be randomised to one of two shoe insert interventions, but will not be informed of the treatment elements or our hypotheses. Trial participants will be unblinded once data analyses have been finalised. Because we are evaluating two different shoe inserts known to have minimal associated adverse events, ${ }^{19}$ it is anticipated that emergency unblinding will not be required.

\section{Interventions}

Forty registered podiatrists and physiotherapists with at least 5 years musculoskeletal experience will fit participants with their allocated intervention. All study practitioners will fit interventions for participants allocated to both groups. To minimise participant burden, study practitioners will be located at multiple private practice clinics across greater Melbourne, Brisbane, Hobart and regional Victoria. To ensure consistency in prescription of foot orthoses and flat shoe inserts, study practitioners will undergo formal training in standardised fitting procedures for both interventions, as used in our previous RCT of prefabricated foot orthoses and flat shoe inserts for young adults with PF pain. ${ }^{19}{ }^{50}$ Study practitioners will also be provided with a comprehensive manual and video outlining study procedures, and will have email and phone access to an unblinded investigator to discuss interventions as required (Melbourne, SEM; Brisbane, BV). Participants will attend an appointment with their study practitioner within 1 week of baseline assessment to undergo fitting of their allocated intervention.

Participants will be asked to wear their allocated inserts as much as possible, and will be able to transfer them 


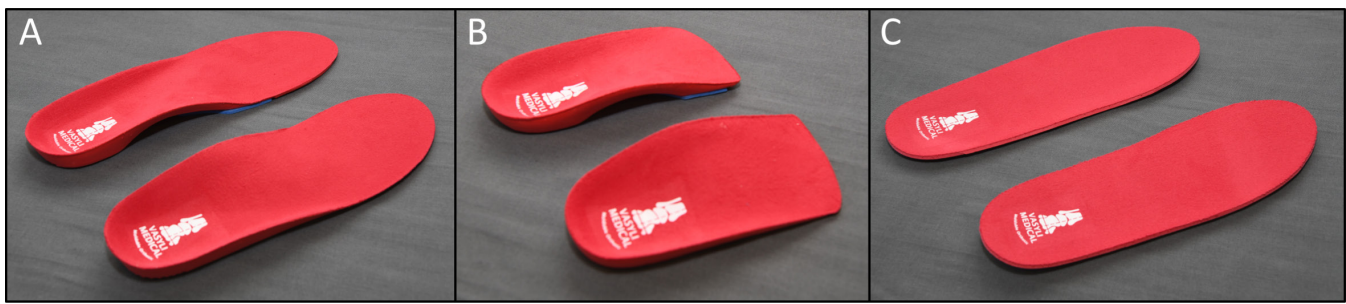

Figure 3 Prefabricated foot orthoses in full length $(A)$ and three-quarter length $(B)$, and flat inserts (C).

between footwear. This reflects current clinical practice and will ensure maximal wear time and potential effects.

\section{Prefabricated foot orthoses}

The prefabricated foot orthoses will replicate the intervention used in our previous RCT in young adults with PF pain. ${ }^{19}{ }^{50}$ Participants will receive prefabricated foot orthoses from a commercially available range (Vasyli Medical, Labrador, Australia) (figure 3A,B). The foot orthoses are manufactured from ethylene-vinyl acetate (EVA) of high density (hard, Shore A $70^{\circ}$ ), medium density $\left(\right.$ Shore A $55^{\circ}$ ) and low density (soft, Shore A $45^{\circ}$ ), and have an inbuilt arch support and $6^{\circ}$ varus wedging. A variety of lengths and shapes are available to fit the shape of different footwear. At their first appointment with their chosen study practitioner, participants will bring up to three pairs of shoes that they most commonly wear (eg, work shoes, casual shoes and sports shoes). Study practitioners will fit one pair of foot orthoses to one pair of the participant's shoes. This will be based on which of the participant's shoes are able to accommodate the foot orthoses and provide the most support, as prefabricated foot orthoses have superior effects when used with supportive footwear. ${ }^{51}$ Where possible, the orthoses will be able to be transferred across their usual footwear. Study practitioners will ensure that the foot orthoses are comfortable, using procedures used in our previous RCT. ${ }^{19} 50$ Figure 4 outlines the steps involved in the prescription algorithm. The first step involves selection of the type and size of orthoses based on shoe volume and foot length, respectively. Step two involves selection of the hardness of the device, based on participant comfort.
If needed, study practitioners will then follow a series of sequential modifications until comfort has been achieved: (1) adding rearfoot varus wedge, (2) adding forefoot varus to the rearfoot varus wedge, (3) removing the rearfoot varus wedge, (4) adding a heel raise and (5) gently heat moulding the orthoses. Comfortable foot orthoses can effectively reduce PF pain in younger adults ${ }^{52}$ and are proposed to optimise adherence and potential therapeutic effects. Participants will be given written instructions for using and adapting to the foot orthoses.

To reflect current clinical practice and to provide sufficient opportunity to ensure adequate comfort and prescribe additional foot orthoses, participants will attend up to six appointments with the study practitioner in the first 6 weeks of the study. Appointments will be scheduled as follows, where appropriate for individual participants: two appointments in week one, one appointment in week two (with an additional appointment in the same week as needed), one appointment in week three or four and one appointment in week six. Participants will be provided with up to four pairs of foot orthoses, fitted to multiple pairs of commonly worn shoes, in order to maximise wear time.

To maximise outcomes of wearing a comfortable, contoured device, participants will receive one pair of sandals (Shore A $50^{\circ}$ ) from the Vionic range (Vionic, Arundel, Queensland, Australia). Participants will be encouraged to wear these during times that they do not normally wear enclosed footwear that accommodates foot orthoses (eg, at home or during warmer weather). Feedback from our previous RCT in young adults with

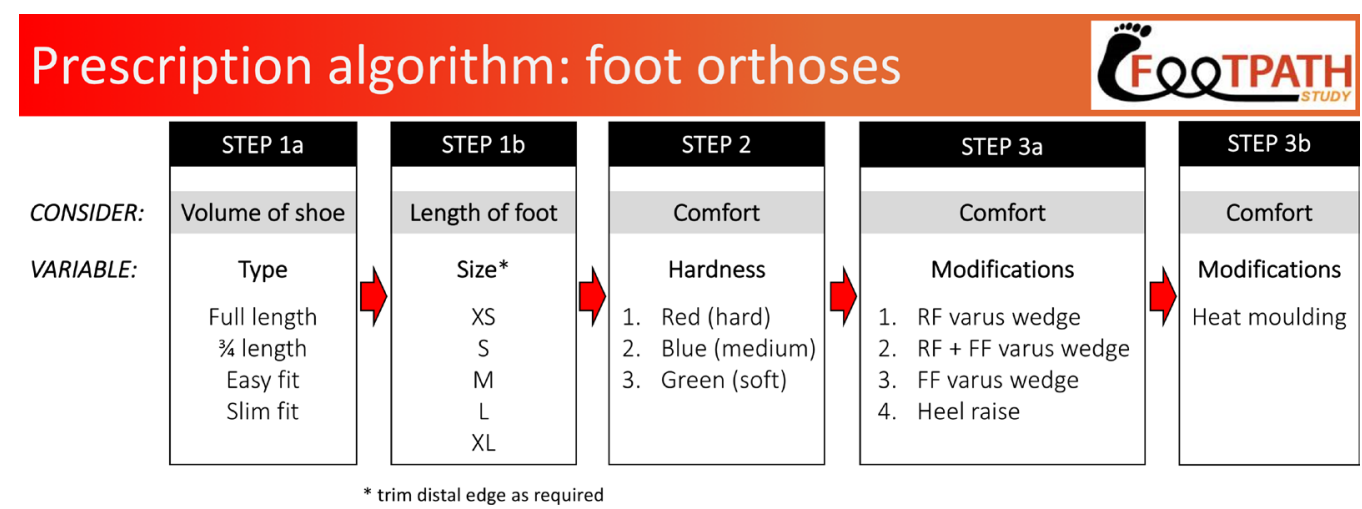

Figure 4 Prescription algorithm for fitting prefabricated foot orthoses. Steps 1 to 3 are to be followed sequentially. Numbered options within each variable are to be trialled sequentially (eg, red orthoses, then blue orthoses, then green orthoses). XS, extra small; S, small; M, medium; L, large; XL, extra large; RF, rearfoot; FF, forefoot. 
PF pain ${ }^{19}$ indicated that participants often chose to wear sandal-type footwear in warm weather, for a large proportion of the year. The Vasyli sandals offered as an adjunct to foot orthoses were well received by participants in our previous RCT and increased the time that participants wore a contoured device.

Participants with a high BMI $\left(\geq 30 \mathrm{~kg} / \mathrm{m}^{2}\right)$ will be invited to attend a follow-up appointment at 6 months post-randomisation, to receive new foot orthoses. This will not be necessary for those with a BMI $<30 \mathrm{~kg} / \mathrm{m}^{2}$, as the pressure-redistributing properties of prefabricated foot orthoses are maintained after 12 months.$^{53}$ However, participants will be offered an additional appointment at 6 months and/or 9 months if they are having any issues with the foot orthoses (eg, increase in pain, excessive wear of orthoses).

\section{Flat shoe inserts}

Flat shoe inserts will be used as the comparator intervention (figure 3C). This is because the contour and wedging of the foot orthoses are proposed to exert mechanical effects on the foot and lower limb, which is thought to be the basis for symptom improvement. Participants will be informed that the study aims to compare two different types of shoe inserts. The flat inserts will be described as an intervention designed to enhance sensory feedback, supported by findings from our previous RCT in PF pain, where those who received flat inserts also experienced improvements in pain over 12 months. ${ }^{19}$ The flat inserts will be the same as those used in our previous RCT, with identical covering fabric to the foot orthoses. To control for gradual contouring that occurs with repeated wear of low-density inserts (a limitation of previous studies), the flat inserts will be made of high-density EVA (Shore A $70^{\circ}$ ). Standardised guidelines for fitting and follow-up of the flat inserts will aim to ensure these are perceived as a credible intervention (figure 5). As with the foot orthoses, participants will be provided with up to four pairs of flat inserts fit to multiple pairs of commonly worn shoes. To address the potential influence of therapist contact, those randomised to this group will also attend an initial appointment with a study practitioner for fitting of flat inserts and up to two follow-up appointments to ensure adequate comfort and fit. At 6 months post-randomisation, a follow-up appointment will be made with the study practitioner to issue new flat inserts, to minimise the effects of cumulative contouring with repeated wear.

At the conclusion of the study, if prefabricated foot orthoses are found to be more efficacious than flat inserts, those randomised to the flat insert group will be offered one pair of foot orthoses and one additional appointment with one of the study practitioners at no cost to them.

\section{Criteria for discontinuing or modifying allocated intervention}

The occurrence of adverse events will be monitored throughout the duration of the RCT by study practitioners, participant logbooks and 3-monthly telephone calls to participants. In the event of minor adverse events (eg, rubbing, blisters) associated with either intervention, study practitioners will review the prescribed device and modify accordingly, based on the prescription algorithms described above. This may include replacement of foot orthoses with a softer device. If participants still report discomfort, they will be encouraged to halve their foot orthoses or flat insert wear time for a period of 2 weeks and then gradually increase wear time as tolerated. If comfort is unable to be achieved, the intervention will be ceased, as this reflects current clinical practice.

In the event of a sustained increase in knee pain or aggravation of another area of pain (eg, low back pain), study practitioners will review the prescribed device and modify accordingly, based on the prescription algorithms. If this does not relieve the participant's symptoms immediately, then intervention will be ceased. Participants who cease their allocated intervention will be encouraged to remain in the trial to enable follow-up data collection at all nominated time points.

Strategies for improving and monitoring adherence to interventions Study personnel will maintain regular communication with participants over the study period (eg, email, phone) and will encourage adherence to the interventions at each

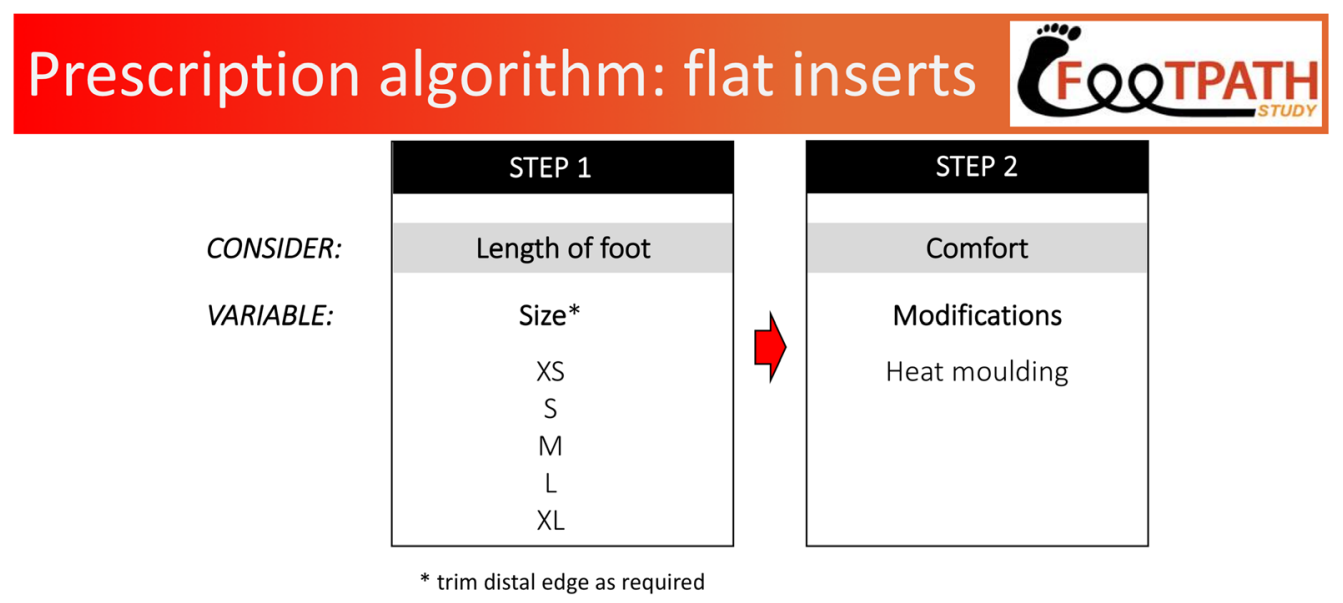

Figure 5 Prescription algorithm for fitting flat inserts. XS, extra small; S, small; M, medium; L, large; XL, extra large. 
time of contact. Adherence to foot orthoses or flat insert wear will be monitored using a variety of strategies. Study practitioners will record attendance at each appointment. To reduce participant burden associated with daily diary entries, participants will report their adherence at 3-monthly intervals during the RCT. This will be recorded as the average days per week and hours per day that they wore the foot orthoses or flat inserts over the preceding 4 weeks. $^{54}$

\section{Concomitant care and interventions}

During the observation and intervention periods, participants will be able to continue with stable medication doses and exercise programmes and use some concomitant interventions (eg, analgesics, heat/cold, general exercise) ${ }^{55}$ New physical therapies (eg, exercise, manual therapy, taping, bracing), intra-articular injections and surgery will be discouraged. If participants have problems with their allocated intervention or wish to seek additional treatment outside the trial, they will be asked to contact the unblinded investigator at their trial site to discuss this (Melbourne, Hobart, JMT/SEM; Brisbane, $\mathrm{BV})$. Use of concomitant interventions will be recorded during the intervention period using monthly logbooks (issued at RCT baseline) and structured questionnaires at 3-monthly intervals.

\section{Participant retention}

Study personnel will use established methods to maximise participant retention. Following enrolment in the study at the commencement of the observation period, participants will be contacted at regular intervals throughout the study period to collect outcome data, ascertain any issues with the intervention and maintain communication. We have endeavoured to minimise participant burden by using an online data collection platform and limiting the number of appointments that participants are required to attend in-person (one screening/baseline appointment, one x-ray appointment and maximum of seven practitioner appointments over 12 months). Although financial incentives will not be provided, financial reimbursement for travel costs will be available for participants if required. Participants who discontinue use of the intervention will be encouraged to complete outcome measures for the duration of the study to minimise missing data.

\section{Outcomes}

Outcome assessment will occur at 3, 6, 9 and 12 months. Three months is the a priori primary end-point of interest, as early improvement in symptoms is likely to influence ongoing adherence with foot orthoses or flat inserts. Twelve-month follow-up will evaluate longer-term effects and economic efficiency of foot orthoses, which is important given the chronic nature of PFOA, and reflects clinical practice.

At entry into the study, participants will be asked their preferred method of receiving and completing outcome measures. Where possible, outcome data will be collected using an internet-based platform, which has equivalent measurement properties to paper-based completion. ${ }^{25}$ This strategy was used in our pilot studies on people with PFOA,${ }^{56}$ ensuring feasibility of online data collection in this population. However, for participants who do not have internet access or would prefer to complete outcome measures in paper format, paper versions and reply-paid envelopes will be mailed.

Outcome measures of GROC, pain and function have been selected based on international recommendations for knee OA. ${ }^{57}$ These are listed below and detailed in online supplementary file 2 .

We have selected patient-reported outcomes over imaging and surgical endpoints, aligning with international recommendations highlighting the importance of the patient-centred outcomes. ${ }^{2257}$ Considering the financial cost and participant burden of repeated MRI and the lack of correlation between symptoms and imaging, ${ }^{58}$ it is important to first determine whether prefabricated foot orthoses improve pain and function. This will ensure continued adherence and greater potential for longer-term effects on joint structure. While total knee replacement is usually recommended for end-stage joint disease, severe pain and functional limitations, ${ }^{755}$ other factors such as race, ethnicity, socioeconomic status and the patient preferences can also influence decisions for surgery. ${ }^{59}$ Thus, patient-reported outcomes are, at present, the ideal method to evaluate foot orthoses outcomes for PFOA. Indeed, regulatory agencies such as the US Food and Drug Administration require the use of patient-reported outcomes in the development of medical products to support labelling claims. ${ }^{60}$

\section{Primary outcome (3 months)}

Knee pain is the predominant symptom of PFOA and the primary indication for undergoing total knee replacement. ${ }^{755}$ Pain will be evaluated as worst knee pain severity during a self-nominated aggravating activity in the previous week. ${ }^{810}$ Participants will nominate one of three everyday activities that they experience the greatest pain severity (rising from sitting, squatting or stair ambulation). Pain severity will be measured on a $100 \mathrm{~mm}$ VAS (terminal descriptors $0=$ no pain and $100=$ worst pain possible). VAS measures of pain severity have well-established reliability and validity, including in PF pain. ${ }^{27}$ This will be measured at baseline, 6 weeks, 3 months (time of primary interest) and 6, 9 and 12 months.

\section{Secondary outcomes}

Secondary outcomes will be administered at baseline, 6 weeks (knee pain severity and GROC), 3 months, 6 and 9 months (knee pain severity and EQ-5D-5L) and 12 months (figure 2).

- Knee pain severity over the past week (100 mm VAS) ${ }^{27}$

- GROC (7-point Likert Scale: 'much better, 'better', 'a little better', 'same', 'a little worse', 'worse' and 'much worse', dichotomised to 'improved' ('much 
better, 'better') versus 'not improved' ('a little better' to 'much worse').

- KOOS subscales: symptoms, pain, function in daily activities, function in sport/recreation, knee-related quality of life and patellofemoral symptoms. ${ }^{30} 31$

- AKPS $^{32}$

- SF-12. ${ }^{33}$

- EQ-5D-5L. ${ }^{34}$

- Tampa Scale for Kinesiophobia. ${ }^{35}$

- Arthritis Self Efficacy Scale. ${ }^{37}$

- Use of co-interventions for knee pain. ${ }^{61}$

Physical activity will be monitored in the subgroup of participants who received a Fitbit physical activity monitor during the observation period. Data relating to physical activity levels (eg, steps, distance) will be extracted weekly for each participant, for the first 3 months after randomisation. Data will be analysed in Microsoft Excel (Microsoft Corporation, Redmond, WA, USA) for total step count and time spent in low, moderate and high-intensity activity bands (defined as step count per $15 \mathrm{~min}$ epoch). All physical activity data will be remotely downloaded from the Fitbit data server using a freely available $\mathrm{R}$ package (Fitbit Scraper) and imported into Microsoft Excel for analysis.

\section{Other outcomes}

Treatment adherence and adverse events: Every 3 months, participants will complete a short questionnaire for physical activity, footwear worn and foot orthoses or flat insert wear time and adverse events. ${ }^{54}$ Evaluation of 12 month adherence is vital to determine whether frequency of wear is maintained long-term. This will assist with translating outcomes into clinical practice guidelines. Study practitioners will record attendance, prescription notes and adverse effects during fitting and follow-up.

Treatment credibility and expectations: The CEQ will be completed again at 3 and 12 months. ${ }^{49}$

\section{Economic outcomes}

Data on direct health costs will be sourced from Medicare and Pharmaceutical Benefits Scheme databases. Direct and indirect health costs (eg, medication use, hospital admissions, other co-interventions such as physiotherapy, time off work due to PFOA or treatment) will be captured from the following sources: (1) monthly participant logbooks, (2) 3-monthly telephone interviews and (3) the Institute for Medical Technology Assessment Productivity Costs Questionnaire. ${ }^{62}$ The EQ-5D is a reliable and valid measure of health-related quality of life, and considers mobility, self-care, usual activity, pain/distress and depression/anxiety. ${ }^{3463}$ EQ-5D will be measured at baseline, and at 3, 6, 9 and 12 months, and used to calculate quality-adjusted life years.

\section{Long-term follow-up}

After completion of the RCT 12 month follow-up, participants will be asked to complete the same battery of patient-reported outcome measures at yearly intervals, up to 4 years after completion of the RCT ( 5 years from baseline). This will allow us to conduct prognostic analyses to identify pain trajectories and predictors of long-term outcome, as in our previous RCTs. ${ }^{64}$

\section{Sample size}

Treatment efficacy will be evaluated by between-group comparisons on the primary outcome measure, which is worst knee pain severity during a self-nominated aggravating activity in the previous week, measured on a $100 \mathrm{~mm}$ VAS. The minimal clinically important difference for pain on a VAS is $15 \mathrm{~mm} .{ }^{65}$ Sample size calculations are based on an analysis of covariance adjusting for baseline of the outcome variable, and assume a between-person SD of $30 \mathrm{~mm}$ (based on pilot data in people with PFOA) and baseline to 3 month correlation of 0.5 . A sample of 160 (80 per group) provides a minimum $90 \%$ power $(\alpha=0.05)$ to detect significant between-group differences and allows for $\sim 20 \%$ dropouts.

Observation period: In people with chronic knee pain, pain severity has been shown to improve naturally over 3 months when people are being monitored by a general practitioner. ${ }^{66}$ Thus, to ensure that participants in the RCT have sufficient levels of pain at baseline, and that any observed improvements in pain during the 3 month time of primary interest are attributable to the intervention, we will include a 3 month observation period prior to randomisation. Based on previous findings, it is anticipated that some participants will experience natural improvement in their pain severity during this time. ${ }^{66}$ Thus, we will continue to recruit participants into the observation period until we have recruited the required sample size into the RCT $(n=160)$. Based on conservative estimates that approximately two thirds of participants will qualify for the RCT at 3 months, it is anticipated that a total of $\sim 230$ participants will be recruited. This will be revised throughout the study period.

\section{Data management and storage}

The majority of outcome data will be collected electronically, facilitating simultaneous data entry. For paperbased data collection, data will be entered by a single trained investigator (JWD). A second investigator will check a random subset of manually entered documents to ensure accuracy. Once data entry is finalised, quality checks will ensure that all data points are within expected values. Only named investigators will have access to the full dataset.

Personal data, including informed consent forms, participant names, contact details and date of birth will be stored on a password-locked computer hard drive, separately from patient-reported or other study data, in order to ensure data de-identification. All subsequent study data will be identified by participant number only, and will be stored on the La Trobe University server Research Data Storage, which is only accessible only by the research team through secure means. All project documentation will be stored on a secure, password locked external hard 
drive, overseen by an external company (DS PRIMA, Port Melbourne, Victoria, Australia). No persons external to the research team will have access to information stored on this server. Appropriate ethical procedures will be followed for all data (eg, participant coding, data file encryption, storage in locked filing cabinets). Any paper containing participant details, such as baseline questionnaires, will remain in the locked filing cabinet and will not be accessible outside the premises. Data pertaining to participant characteristics, questionnaires and clinical tests will be preserved for possible future use by the investigators. De-identified data will be stored in an Excel spreadsheet. If researchers other than those listed as investigators wish to use the data, prior approval will be sought from the La Trobe University Human Ethics Committee. Participants will be made aware of this in the participant information statement, ensuring that they are aware of the possibility that their data will be used for future studies and are able to provide written informed consent.

Due to the minimal known risks associated with the interventions being evaluated, this study will not require a formal data monitoring committee or planned interim analysis.

\section{Statistical methods}

\section{Primary and key secondary objectives}

Intention to treat analyses will be performed, with all randomised participants included regardless of protocol adherence. Blinded analyses of primary and secondary patient-reported outcomes will be performed. The dichotomised measure of GROC will be expressed from blinded analyses as relative risk and number needed to treat, with $95 \% \mathrm{CI}$, to facilitate clinical guidelines. ${ }^{1967}$ For the primary outcome and continuous secondary outcome measures, linear mixed models (with baseline value as a covariate and treatment condition as a fixed factor) will be used to evaluate the treatment effect and $95 \%$ CI at 3 months and 12 months $(p<0.05)$. Linear mixed models utilising repeated measures at all time-points will allow non-biased estimates of treatment effect in the presence of any potential missing cases. This likelihood-based estimation procedure results in non-biased estimates, providing data are missing at random and models are adjusted for any imbalance between groups in potential confounders at baseline (age, sex, weight, symptom duration, PF/TF OA radiographic severity). Relative risk (95\% CIs) will be calculated for use of co-interventions and adverse events.

\section{Economic evaluation}

Blinded economic analyses will be conducted to evaluate the 12 month economic efficiency of prefabricated foot orthoses compared with flat shoe inserts, from the societal perspective. Hospitalisations will be converted to costs using the National Weighted Activity Unit costing model. Incremental cost-effectiveness analyses will use the formula $\left(\left[\mathrm{DC}_{\text {foot orthoses }}+\mathrm{IC}_{\text {foot orthoses }}\right]-\left[\mathrm{DC}_{\text {flat inserts }}+\right.\right.$ $\left.\left.\mathrm{IC}_{\text {flat inserts }}\right] /\left[\mathrm{E}_{\text {foot orthoses }}-\mathrm{E}_{\text {flat inserts }}\right]\right)$, where $\mathrm{DC}=$ mean direct health costs, $\mathrm{IC}=$ mean indirect costs, $\mathrm{E}=\mathrm{effect}$, foot orthoses $=$ foot orthoses group and ${ }_{\text {flat inserts }}=$ flat insert group. Effect for the primary economic evaluation will be the proportion of participants who 'improve' (measured on the GROC) within each group at 12 months. Thus, the cost-effectiveness ratio will reflect the marginal cost per additional 'improved' participant from the societal perspective over a 12 month time horizon. Uncertainty in this ratio will be examined by constructing a $95 \%$ confidence ellipse on a cost-effectiveness plane and transforming these to cost-effectiveness acceptability curves using non-parametric bootstrap resampling of primary data. Sensitivity analyses will be conducted, varying the threshold of 'improvement' on the GROC to reflect increasingly higher thresholds. Quality-adjusted life year scores for each participant (calculated as area under the curve applied to utility measures calculated from EQ-5D) will be substituted for GROC scores as the effect measure, creating an incremental cost-utility ratio to determine the marginal cost per additional quality-adjusted life year for the more effective intervention.

\section{PATIENT AND PUBLIC INVOLVEMENT}

Patients and the public were not directly involved in the development of the research question, study design or selection of outcome measures. Patients will not be directly involved in the recruitment to or conduct of the study, except as participants if they meet the eligibility criteria and provide informed consent. At the conclusion of the study, overall study findings and individual participant data will be provided to study participants on request.

\section{ETHICS AND DISSEMINATION}

This study complies with the Declaration of Helsinki and has been approved by ethics committees at La Trobe University and The University of Queensland. All participants will provide written informed consent prior to baseline data collection and enrolment in the 3 month observation period. Participant information and consent forms for each phase of the study are included in online supplementary file 1. Participants will undergo knee radiographs at a single time point as part of this trial, ensuring that the amount of ionising radiation is consistent with standard clinical exposure. When prescribed by trained health practitioners, prefabricated foot orthoses and flat shoe inserts are associated with minimal and transient adverse events. ${ }^{19}$ Thus, there are minimal ethical and safety considerations associated with this trial.

Study outcomes will be widely disseminated through a variety of sources. Primary and key secondary objectives will be submitted to a high-impact peer-reviewed journal in the field. Because study outcomes are applicable to a broad range of health professionals, we will target a general medical journal to facilitate wider dissemination of findings to key stakeholders (eg, general practitioners). 
Each of the other secondary objectives will be addressed in separate publications and submitted to appropriate journals in the field. Authorship will be in accordance with guidelines provided by the International Committee of Medical Journal Editors. ${ }^{68}$ We will also submit articles to key professional magazines to enhance dissemination to clinicians. Our publication strategy will be complemented by submission of abstracts to key national and international conferences, covering multiple discipline groups (eg, physiotherapy, podiatry, general practice) as well as OA conferences. We will also develop an open access website and resources for clinicians, including videos detailing how to prescribe foot orthoses, and run workshops on PFOA and foot orthoses for registered health professionals. This will facilitate translation of findings to clinical practice, especially practitioners located in rural or remote areas.

\section{DISCUSSION}

PFOA is a major public health problem and has no cure. Pain and stiffness experienced during daily activities, occupational tasks and exercise can reduce active participation. Importantly, PFOA in middle-aged adults can affect productivity and contribution to society and result in more years of knee pain and disability across the lifespan. Along with direct personal and economic costs of PFOA, indirect costs associated with consequences of physical inactivity are a major burden on health expenditure.

This RCT will be the first to evaluate patient-reported benefits of foot orthoses - a simple, low-cost, low-risk intervention that is widely accessible to people with PFOA. Findings of efficacy and cost-effectiveness of prefabricated foot orthoses could represent a turning point in the effective long-term management of PFOA. When worn in everyday and exercise footwear, foot orthoses have the potential to reduce pain every time the foot hits the ground, substantially increasing an individual's capacity and motivation to be physically active. This has important implications for maintenance of general and mental health with increasing age. Importantly, the ease of daily use of foot orthoses, with minimal patient burden, is likely to maximise adherence, enhance outcomes and reduce reliance on health practitioner resources.

\section{Author affiliations}

${ }^{1}$ Division of Physiotherapy, School of Health and Rehabilitation Sciences, The University of Queensland, Brisbane, Queensland, Australia

${ }^{2}$ La Trobe Sport and Exercise Medicine Research Centre, College of Science, Health and Engineering, La Trobe University, Melbourne, Victoria, Australia

${ }^{3}$ Discipline of Podiatry, School of Allied Health, College of Science, Health and

Engineering, La Trobe University, Melbourne, Victoria, Australia

${ }^{4}$ School of Physiotherapy and Exercise Science, Curtin University, Perth, Western Australia, Australia

${ }^{5}$ Centre for Health, Exercise and Sports Medicine, Department of Physiotherapy, School of Health Sciences, University of Melbourne, Melbourne, Victoria, Australia ${ }^{6}$ School of Primary and Allied Health Care, Monash University, Melbourne, Victoria, Australia
${ }^{7}$ School of Physical Therapy and Bone and Joint Institute, University of Western Ontario, London, Ontario, Canada

Contributors NJC, KMC, HBM, TGR, AJS, RSH, BV and TPH conceived the study and obtained funding. NJC, KMC and HBM designed the trial protocol with input from TGR, AJS, RSH, BV, TPH, SEM and JMT. AJS and TPH provided statistical expertise. AJS will conduct primary statistical analysis. NJC drafted the manuscript with input from KMC, HBM, TGR, AJS, RSH, BV, TPH, SEM, JMT, HFH, BEP, GC, JWD and LRM. All authors have read and approved the final manuscript.

Funding This work is supported by the National Health and Medical Research Council (Australia) (ID: 1106852; 2016-2019). NJC previously held a University of Queensland Postdoctoral Research Fellowship (2015-2017). HBM is currently a National Health and Medical Research Council Senior Research Fellow (ID: 1135995). RSH currently holds an Australian Research Council (ARC) Future Fellowship (ID: FT130100175). Vasyli Medical (Labrador, Australia) will provide the foot orthoses, flat inserts and sandals.

Competing interests BV reports non-financial support from Vionic, outside the submitted work. He is a member (non-paid affiliation) of the Vasyli Think Tank, which was founded in 2011 to foster collaboration and cooperative thought among a leading group of health professionals specialising in the field of lower limb biomechanics

\section{Patient consent for publication Not required.}

Ethics approval Ethical approval has been granted by La Trobe University's Human Ethics Committee (HEC16-113) and The University of Queensland's Medical Research Ethics Committee (2017000284).

Provenance and peer review Not commissioned; externally peer reviewed.

Open access This is an open access article distributed in accordance with the Creative Commons Attribution Non Commercial (CC BY-NC 4.0) license, which permits others to distribute, remix, adapt, build upon this work non-commercially, and license their derivative works on different terms, provided the original work is properly cited, appropriate credit is given, any changes made indicated, and the use is non-commercial. See: http://creativecommons.org/licenses/by-nc/4.0/.

\section{REFERENCES}

1. Hinman RS, Lentzos J, Vicenzino B, et al. Is patellofemoral osteoarthritis common in middle-aged people with chronic patellofemoral pain? Arthritis Care Res 2014;66:1252-7.

2. Duncan RC, Hay EM, Saklatvala J, et al. Prevalence of radiographic osteoarthritis-it all depends on your point of view. Rheumatology 2006:45:757-60.

3. Duncan R, Peat G, Thomas E, et al. Incidence, progression and sequence of development of radiographic knee osteoarthritis in a symptomatic population. Ann Rheum Dis 2011;70:1944-8.

4. Kornaat PR, Bloem JL, Ceulemans RY, et al. Osteoarthritis of the knee: association between clinical features and MR imaging findings. Radiology 2006;239:811-7.

5. Hunter DJ, March L, Sambrook PN. The association of cartilage volume with knee pain. Osteoarthr Cartil 2003;11:725-9.

6. Hendry M, Williams NH, Markland D, et al. Why should we exercise when our knees hurt? A qualitative study of primary care patients with osteoarthritis of the knee. Fam Pract 2006;23:558-67.

7. Carr AJ, Robertsson O, Graves S, et al. Knee replacement. Lancet 2012;379:1331-40

8. Crossley KM, Vicenzino B, Lentzos J, et al. Exercise, education, manual-therapy and taping compared to education for patellofemoral osteoarthritis: a blinded, randomised clinical trial. Osteoarthr Cartil 2015;23:1457-64.

9. Quilty B, Tucker M, Campbell R, et al. Physiotherapy, including quadriceps exercises and patellar taping, for knee osteoarthritis with predominant patello-femoral joint involvement: randomized controlled trial. J Rheumatol 2003;30:1311-7.

10. Callaghan MJ, Parkes MJ, Hutchinson CE, et al. A randomised trial of a brace for patellofemoral osteoarthritis targeting knee pain and bone marrow lesions. Ann Rheum Dis 2015;74:1164-70.

11. Campbell R, Evans M, Tucker M, et al. Why don't patients do their exercises? Understanding non-compliance with physiotherapy in patients with osteoarthritis of the knee. J Epidemiol Community Health 2001:55:132-8.

12. Squyer E, Stamper DL, Hamilton DT, et al. Unloader knee braces for osteoarthritis: do patients actually wear them? Clin Orthop Relat Res 2013;471:1982-91. 
13. Crossley KM, van Middelkoop M, Callaghan MJ, et al. 2016 Patellofemoral pain consensus statement from the 4th International Patellofemoral Pain Research Retreat, Manchester. Part 2 recommended physical interventions (exercise, taping, bracing, foot orthoses and combined interventions). Br J Sports Med 2016;50:844-52.

14. Barton CJ, Lack S, Hemmings S, et al. The 'Best Practice Guide to Conservative Management of Patellofemoral Pain': incorporating level 1 evidence with expert clinical reasoning. Br J Sports Med 2015;49:923-34.

15. Crossley KM, Hinman RS. The patellofemoral joint: the forgotten joint in knee osteoarthritis. Osteoarthr Cartil 2011:19:765-7.

16. Wyndow N, Collins N, Vicenzino B, et al. Is there a biomechanical link between patellofemoral pain and osteoarthritis? A narrative review. Sports Med 2016;46:1797-808.

17. Crossley KM, Schache AG, Ozturk H, et al. Pelvic and hip kinematics during walking in people with patellofemoral joint osteoarthritis compared to healthy age-matched controls. Arthritis Care Res 2018;70:309-14.

18. Collins NJ, Hinman RS, Menz HB, et al. Immediate effects of foot orthoses on pain during functional tasks in people with patellofemoral osteoarthritis: a cross-over, proof-of-concept study. Knee 2017:24:76-81.

19. Collins N, Crossley K, Beller E, et al. Foot orthoses and physiotherapy in the treatment of patellofemoral pain syndrome: randomised clinical trial. BMJ 2008;337:a1735

20. Chan AW, Tetzlaff JM, Altman DG, et al. SPIRIT 2013 statement: defining standard protocol items for clinical trials. Ann Intern Med 2013;158:200-7.

21. Chan AW, Tetzlaff JM, Gøtzsche PC, et al. SPIRIT 2013 explanation and elaboration: guidance for protocols of clinical trials. BMJ 2013;346:e7586.

22. McAlindon TE, Driban JB, Henrotin Y, et al. OARSI Clinical Trials Recommendations: Design, conduct, and reporting of clinical trials for knee osteoarthritis. Osteoarthr Cartil 2015;23:747-60.

23. van Middelkoop M, Bennell KL, Callaghan MJ, et al. International patellofemoral osteoarthritis consortium: consensus statement on the diagnosis, burden, outcome measures, prognosis, risk factors and treatment. Semin Arthritis Rheum 2018:47:666-75.

24. National Institute for Health and Care Excellence. Osteoarthritis: care and management. 2014. https://www.nice.org.uk/guidance/cg177 (accessed 8 Jul 2018).

25. Gudbergsen H, Bartels EM, Krusager P, et al. Test-retest of computerized health status questionnaires frequently used in the monitoring of knee osteoarthritis: a randomized crossover trial. BMC Musculoskelet Disord 2011:12:190.

26. de Klerk BM, Willemsen S, Schiphof D, et al. Development of radiological knee osteoarthritis in patients with knee complaints. Ann Rheum Dis 2012;71:905-10.

27. Crossley KM, Bennell KL, Cowan SM, et al. Analysis of outcome measures for persons with patellofemoral pain: which are reliable and valid? Arch Phys Med Rehabil 2004;85:815-22.

28. Freynhagen R, Baron R, Gockel U, et al. painDETECT: a new screening questionnaire to identify neuropathic components in patients with back pain. Curr Med Res Opin 2006;22:1911-20.

29. Boudreau SA, Kamavuako EN, Rathleff MS. Distribution and symmetrical patellofemoral pain patterns as revealed by highresolution 3D body mapping: a cross-sectional study. BMC Musculoskelet Disord 2017;18:160.

30. Roos EM, Roos HP, Lohmander LS, et al. Knee Injury and Osteoarthritis Outcome Score (KOOS)-development of a selfadministered outcome measure. J Orthop Sports Phys Ther 1998;28:88-96

31. Crossley KM, Macri EM, Cowan SM, et al. The patellofemoral pain and osteoarthritis subscale of the KOOS (KOOS-PF): development and validation using the COSMIN checklist. Br J Sports Med 2018:52:1130-6.

32. Kujala UM, Jaakkola LH, Koskinen SK, et al. Scoring of patellofemoral disorders. Arthroscopy 1993;9:159-63.

33. Ware J, Kosinski M, Keller SD. A 12-Item Short-Form Health Survey: construction of scales and preliminary tests of reliability and validity. Med Care 1996;34:220-33.

34. Rabin R, de Charro F. EQ-5D: a measure of health status from the EuroQol Group. Ann Med 2001;33:337-43.

35. Kori SH, Miller RP, Todd DD. Kinisiophobia: a new view of chronic pain behaviour. Pain Management 1990:35-43.

36. Sullivan MJL, Bishop SR, Pivik J. The Pain Catastrophizing Scale: development and validation. Psychol Assess 1995;7:524-32.

37. Lorig K, Chastain RL, Ung E, et al. Development and evaluation of a scale to measure perceived self-efficacy in people with arthritis. Arthritis Rheum 1989;32:37-44.
38. Schiphof D, van Middelkoop M, de Klerk BM, et al. Crepitus is a first indication of patellofemoral osteoarthritis (and not of tibiofemoral osteoarthritis). Osteoarthr Cartil 2014;22:631-8.

39. Redmond AC, Crosbie J, Ouvrier RA. Development and validation of a novel rating system for scoring standing foot posture: the Foot Posture Index. Clin Biomech 2006;21:89-98.

40. McPoil TG, Vicenzino B, Cornwall MW, et al. Reliability and normative values for the foot mobility magnitude: a composite measure of vertical and medial-lateral mobility of the midfoot. J Foot Ankle Res 2009;2:6.

41. Bennell KL, Talbot RC, Wajswelner $\mathrm{H}$, et al. Intra-rater and inter-rater reliability of a weight-bearing lunge measure of ankle dorsiflexion. Aust J Physiother 1998;44:175-80.

42. Barton CJ, Bonanno D, Menz HB. Development and evaluation of a tool for the assessment of footwear characteristics. J Foot Ankle Res 2009;2:10.

43. Lord SR, Menz HB, Tiedemann A. A physiological profile approach to falls risk assessment and prevention. Phys Ther 2003;83:237-52.

44. Peters DM, Fritz SL, Krotish DE. Assessing the reliability and validity of a shorter walk test compared with the 10-Meter Walk Test for measurements of gait speed in healthy, older adults. J Geriatr Phys Ther 2013;36:24-30.

45. Kellgren JH, Jeffrey MR, Ball J. The Epidemiology of Chronic Rheumatism: Atlas of Standard Radiographs. Oxford: Blackwell Scientific, 1963.

46. Altman RD, Hochberg M, Murphy WA, et al. Atlas of individual radiographic features in osteoarthritis. Osteoarthr Cartil 1995;3(Suppl A):3-70

47. Moyer R, Wirth W, Duryea J, et al. Anatomical alignment, but not goniometry, predicts femorotibial cartilage loss as well as mechanical alignment: data from the Osteoarthritis Initiative. Osteoarthr Cartil 2016;24:254-61.

48. Macri EM, Stefanik JJ, Khan KK, et al. Is tibiofemoral or patellofemoral alignment or trochlear morphology associated with patellofemoral osteoarthritis? A systematic review. Arthritis Care Res 2016;68:1453-70.

49. Devilly GJ, Borkovec TD. Psychometric properties of the credibility/expectancy questionnaire. J Behav Ther Exp Psychiatry 2000;31:73-86.

50. Vicenzino B, Collins N, Crossley K, et al. Foot orthoses and physiotherapy in the treatment of patellofemoral pain syndrome: a randomised clinical trial. BMC Musculoskelet Disord 2008;9:27.

51. Barton CJ, Menz HB, Crossley KM. Clinical predictors of foot orthoses efficacy in individuals with patellofemoral pain. Med Sci Sports Exerc 2011;43:1603-10.

52. Mills K, Blanch P, Dev P, et al. A randomised control trial of short term efficacy of in-shoe foot orthoses compared with a wait and see policy for anterior knee pain and the role of foot mobility. Br J Sports Med 2012;46:247-52.

53. Cronkwright DG, Spink MJ, Landorf KB, et al. Evaluation of the pressure-redistributing properties of prefabricated foot orthoses in older people after at least 12 months of wear. Gait Posture 2011;34:553-7.

54. Munteanu SE, Landorf KB, McClelland JA, et al. Shoe-stiffening inserts for first metatarsophalangeal joint osteoarthritis (the SIMPLE trial): study protocol for a randomised controlled trial. Trials 2017:18:198.

55. Zhang W, Moskowitz RW, Nuki G, et al. OARSI recommendations for the management of hip and knee osteoarthritis, Part II: OARS evidence-based, expert consensus guidelines. Osteoarthr Cartil $2008 ; 16: 137-62$

56. Wyndow N, Crossley KM, Vicenzino B, et al. A single-blinded randomized, parallel group superiority trial investigating the effects of footwear and custom foot orthoses versus footwear alone in individuals with patellofemoral joint osteoarthritis: a phase II pilot trial protocol. J Foot Ankle Res 2017;10:19.

57. Bellamy N, Kirwan J, Boers M, et al. Recommendations for a core set of outcome measures for future phase III clinical trials in knee, hip, and hand osteoarthritis. Consensus development at OMERACT III. $J$ Rheumatol 1997;24:799-802.

58. Bedson J, Croft PR. The discordance between clinical and radiographic knee osteoarthritis: a systematic search and summary of the literature. BMC Musculoskelet Disord 2008:9:116.

59. Gossec L, Paternotte S, Maillefert JF, et al. The role of pain and functional impairment in the decision to recommend total joint replacement in hip and knee osteoarthritis: an international cross-sectional study of 1909 patients. Report of the OARSIOMERACT Task Force on total joint replacement. Osteoarthr Cartil 2011;19:147-54

60. U.S. Department of Health and Human Services FDA Center for Drug Evaluation and Research, U.S. Department of Health and 
Human Services FDA Center for Biologics Evaluation and Research, U.S. Department of Health and Human Services FDA Center for Devices and Radiological Health. Guidance for industry: patientreported outcome measures: use in medical product development to support labeling claims: draft guidance. Health Qual Life Outcomes 2006;4:79.

61. Munteanu SE, Scott LA, Bonanno DR, et al. Effectiveness of customised foot orthoses for Achilles tendinopathy: a randomised controlled trial. Br J Sports Med 2015;49:989-94.

62. Bouwmans $\mathrm{C}, \mathrm{Krol}$ M, Severens H, et al. The iMTA Productivity Cost Questionnaire: a standardized instrument for measuring and valuing health-related productivity losses. Value Health 2015;18:753-8.

63. Gignac MA, Cao X, Mcalpine J, et al. Measures of disability: Arthritis Impact Measurement Scales 2 (AIMS2), Arthritis Impact Measurement Scales 2-Short Form (AIMS2-SF), The Organization for Economic Cooperation and Development (OECD) Long-Term Disability (LTD) Questionnaire, EQ-5D, World Health Organization Disability Assessment Schedule II (WHODASII), Late-Life Function and Disability Instrument (LLFDI), and Late-Life Function and
Disability Instrument-Abbreviated Version (LLFDI-Abbreviated). Arthritis Care Res 2011;63(Suppl 11):S308-24.

64. Collins NJ, Bierma-Zeinstra SM, Crossley KM, et al. Prognostic factors for patellofemoral pain: a multicentre observational analysis. Br J Sports Med 2013;47:227-33.

65. Salaffi F, Stancati A, Silvestri CA, et al. Minimal clinically important changes in chronic musculoskeletal pain intensity measured on a numerical rating scale. Eur J Pain 2004;8:283-91.

66. Kastelein M, Luijsterburg PA, Belo JN, et al. Six-year course and prognosis of nontraumatic knee symptoms in adults in general practice: a prospective cohort study. Arthritis Care Res 2011;63:1287-94.

67. Crossley K, Bennell K, Green S, et al. Physical therapy for patellofemoral pain: a randomized, double-blinded, placebocontrolled trial. Am J Sports Med 2002;30:857-65.

68. International Committee of Medical Journal Editors. Uniform requirements for manuscripts submitted to biomedical journals: Writing and editing for biomedical publication. 2010. Available http:// www.icmje.org/recommendations/browse/roles-and-responsibilities/ defining-the-role-of-authors-and-contributors.html (accessed $23 \mathrm{Nov}$ 2017). 